\title{
Stress Hormone Levels in Awake Craniotomy and Craniotomy under General Anesthesia
}

\author{
Nobusada Shinoura $^{1^{*}}$, Ryoji Yamada ${ }^{1}$, Kaoru Hatori ${ }^{2}$, Hiroshi Sato ${ }^{2}$, and Kohei Kimura ${ }^{2}$ \\ ${ }^{1}$ Department of Neurosurgery, Komagome Metropolitan Hospital, Hon-komagome, Bunkyo-ku, Tokyo, Japan \\ ${ }^{2}$ Department of Anesthesiology, Komagome Metropolitan Hospital, Hon-komagome, Bunkyo-ku, Tokyo, Japan
}

"Corresponding author: Nobusada Shinoura, Department of Neurosurgery, Komagome Metropolitan Hospital 3-18-22 Hon-komagome, Bunkyo-ku, Tokyo 113-8677, Japan, Tel: +81-3-3823-2101, Fax: +81-3-3824-1552; E-mail: shinoura@cick.jp

Received date: Nov 19, 2014, Accepted date: Dec 16, 2014, Published date: Dec 20, 2014

Copyright: $\odot 2014$ Shinoura N, et al. This is an open-access article distributed under the terms of the Creative Commons Attribution License, which permits unrestricted use, distribution, and reproduction in any medium, provided the original author and source are credited.

\begin{abstract}
To compare stress levels between awake craniotomy and craniotomy under general anesthesia, we analyzed plasma levels of adrenaline, cortisol, adrenocorticotropic hormone (ACTH), noradrenaline and dopamine in a large series of patients. Patients who underwent awake craniotomy in our hospital $(n=110)$ were evaluated at 5 sample times: immediately after arterial line insertion (T1); immediately after head fixation in a head frame (T2); $1 \mathrm{~h}$ after start of incision (T3); immediately after relief of head fixation (T4); and immediately after arrival in the intensive care unit (T5). Levels were then compared with those in 15 patients who underwent craniotomy under general anesthesia. Plasma levels of adrenaline were significantly higher during awake craniotomy than in craniotomy under general anesthesia at T1 to T4. Plasma levels of ACTH, cortisol, and noradrenaline in craniotomy were significantly higher under general anesthesia than those in awake craniotomy at T5. No correlations were seen between plasma levels of adrenaline in awake craniotomy and age, sex, preoperative Karnofsy Performance Scale score or postoperative neurological status. In conclusion, plasma levels of adrenaline were significantly higher in awake craniotomy than in craniotomy under general anesthesia during surgery, while plasma levels of cortisol, ACTH and noradrenaline were significantly higher in craniotomy under general anesthesia than those in awake craniotomy just after surgery.
\end{abstract}

Keywords: Adrenocorticotropic hormone; Adrenaline; Awake craniotomy; Cortisol; General anesthesia; Noradrenaline, Stress hormone

\section{Introduction}

Awake craniotomy for brain lesions has recently been reported to offer numerous benefits to patients. Awake craniotomy was initially reported to be associated with improved neurological outcomes in functional areas with maximal removal of lesions compared to results under general anesthesia, thanks to the ability to identify eloquent areas during surgery [1-3]. In addition, awake craniotomy has been reported to minimize both intensive care time and total hospital stay [4,5].

Despite the surgical benefits of awake craniotomy for patients, analysis of the surgical stress associated with awake craniotomy compared with craniotomy under general anesthesia seems likely to be important. Several investigators have reported the responses of inflammatory mediators and amino acids during awake craniotomy [6,7], but no such reports have described stress hormone responses during awake craniotomy. The goal of the present study was to compare stress hormone levels during awake craniotomy with those during craniotomy under general anesthesia.

\section{Patients and Methods}

\section{Patients}

Participants in this retrospective study comprised patients who underwent craniotomy between 2008 and 2013 at Komagome Metropolitan Hospital. A total of consecutive 110 patients with regions suspected preoperatively of containing supratentorial brain tumor underwent awake craniotomy. Another 15 patients with regions suspected preoperatively of containing supratentorial brain tumor underwent craniotomy under general anesthesia. The 110 patients who underwent awake craniotomy comprised 59 men and 51 women, with a median age of 59 years at the time of surgery (range, 25-83 years), while the 15 patients with craniotomy under general anesthesia comprised 7 men and 8 women, with a median age of 63 years at the time of surgery (range, 48-82 years).

Informed consent for craniotomy was obtained from all patients prior to surgery.

\section{Awake craniotomy}

Awake craniotomy was performed as described previously [8]. Briefly, patients were positioned in a supine or lateral position with rigid head fixation (Sugita headrest; Mizuho Medical, Tokyo, Japan) after administration of a local anesthetic ( $1 \%$ xylocaine with epinephrine and $0.75 \%$ anapain) at the pin sites and regional field block sites. Under general anesthesia with propofol, dexmedetomidine or remifentanil, the skin was infiltrated with the same local anesthetic 
agent and incised, and neuronavigated craniotomy and incision of the dura was performed.

After suspending administration of the anesthetic agent, oxygen was administered via a mask, and cortical mapping was performed by stimulating the cortex with a modified Ojemann stimulator [9]. To avoid inducing intraoperative seizures, a low-stimulus setting (3-5 $\mathrm{mA}, 60-\mathrm{Hz}$ biphasic square wave pulse of $1 \mathrm{~ms} /$ phase for $4 \mathrm{~s}$ ) was used. The tumor was removed in the usual fashion. Adequacy of function was continuously assessed during tumor removal [8]. In the languagerelated area, language functions such as object naming, auditory comprehension, repetition, reading and writing were continuously evaluated. In the motor-related area, motor functions such as tongue movement, eye closing, hand clenching, elbow flexion, knee flexion, and foot flexion were continuously checked. In the sensory-related area, sensory functions such as tactile perception and deep sensation were continuously evaluated. In the non-dominant hemisphere, memory was continuously checked, including forward and backward counting, the frontal assessment battery (FAB) for patients with tumors within the frontal lobes, word and visual memory, and recognition of facial expressions for patients with tumors within the temporal lobes [10]. Tumor removal was assisted by a neuronavigation system (Stealth; Medtronic Sofamor Danek, Osaka, Japan). If neurological deficit occurred at any point during the resection, the operation was interrupted, and neurological function was assessed over the next $5 \mathrm{~min}$. If the neurological deficit did not recover, the operation was terminated. Following completion of tumor resection, intravenous anesthesia was administered using dexmedetomidine or propofol. After closure of the dura, the bone flap was replaced, and the skin was closed in the usual manner.

\section{Craniotomy under general anesthesia}

Craniotomy under general anesthesia was performed as follows. Patients were placed in a supine or lateral position with rigid head fixation (Sugita headrest; Mizuho Medical). The skin was incised, and neuronavigated craniotomy and incision of the dura were performed. Anesthesia was maintained with $50 \%$ oxygen in air and $3.5 \%$ desflurane, remifentanil at $0.1-0.3 \mu \mathrm{g} / \mathrm{kg} / \mathrm{min}$, propofol at $3-4 \mathrm{mg} / \mathrm{kg} / \mathrm{h}$, a fentanyl bolus of $100 \mu \mathrm{g}$, and rocuronium at $0.3-0.6 \mathrm{mg} / \mathrm{kg} / \mathrm{h}$ was given as needed. The tumor was removed in the usual fashion. Tumor removal was assisted by a neuronavigation system. Following completion of tumor resection, the dura was closed, the bone flap was replaced, and the skin was closed in the usual manner.

\section{Sampling during and after craniotomy}

Sample times in awake craniotomy and craniotomy under general anesthesia were as follows: T1, immediately after arterial line insertion; T2, immediately after head was fixed by head frame; T3, $1 \mathrm{~h}$ after start of incision; T4, immediately after relief of head fixation; and T5, immediately after arrival in the intensive care unit. At each sample time, $4 \mathrm{ml}$ of blood was withdrawn to determine levels of adrenaline, noradrenaline, cortisol, adrenocorticotropic hormone (ACTH) and dopamine. Concentrations of those hormones were analyzed externally by SRL (Tokyo, Japan). Normal ranges of those hormones according to SRL norms were as follows: adrenaline, $<100 \mathrm{pg} / \mathrm{ml}$; noradrenaline, $100-450 \mathrm{pg} / \mathrm{ml}$; cortisol, $4-18.3 \mu \mathrm{g} / \mathrm{dl}$; ACTH, 7.2-63.3 $\mathrm{pg} / \mathrm{ml}$; and dopamine, $<20 \mathrm{pg} / \mathrm{ml}$.

\section{Statistical analysis}

The Wilcoxon rank-sum test was used to compare stress hormone levels between awake craniotomy and craniotomy under general anesthesia, and mean adrenaline levels at the 5 sample times between men and women and between Karnofsy Performance Scale (KPS) score before surgery 80 (Group A) and $<80$ (Group B). Correlations between mean adrenaline levels at the 5 sample times and age were analyzed using Spearman's rank correlation. Correlations of mean plasma levels of adrenaline at the 5 sample times among patients showing deterioration, no change or improvement of neurological status after awake craniotomy were analyzed using the Kruskal-Wallis rank-sum test. All $\mathrm{p}$-values were two-sided and values of $\mathrm{p}<0.05$ were considered statistically significant. All statistical analyses were performed with EZR (Saitama Medical Centre, Jichi Medical University; http://www.jichi.ac.jp/saitama-sct/SaitamaHP.files/ statmedEN.html;[11]), a graphical user interface for R version 2.13.0 (The R Foundation for Statistical Computing, Vienna, Austria). More precisely, EZR is a modified version of $\mathrm{R}$ Commander version 1.6-3 that was designed to add statistical functions frequently used in biostatistics.

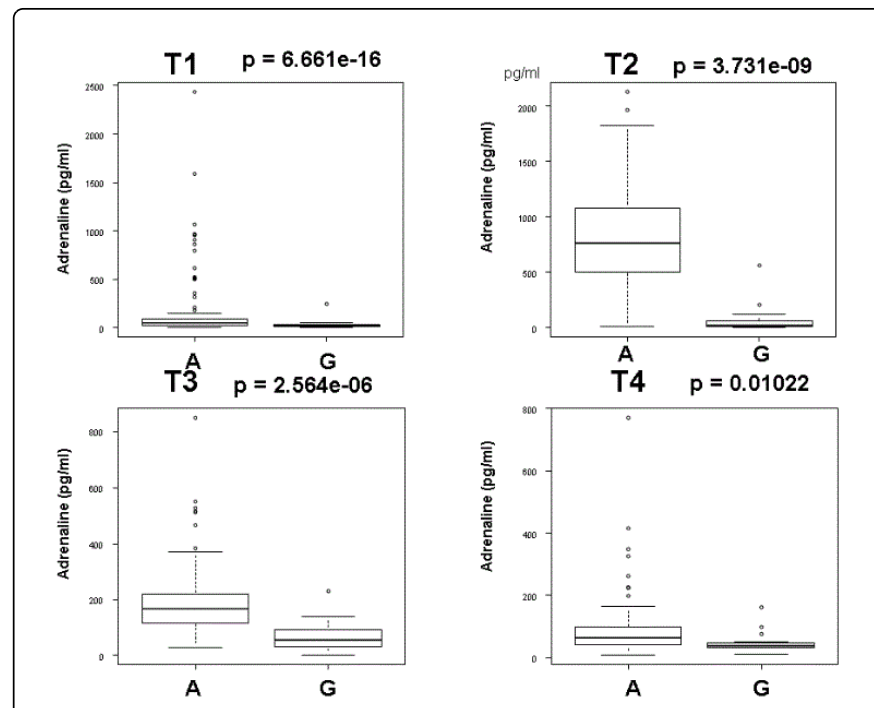

Figure 1: Comparison of plasma levels of adrenaline between awake craniotomy and craniotomy under general anesthesia Plasma levels of adrenaline in awake craniotomy and craniotomy under general anesthesia were compared using the Wilcoxon rank-sum test. A, awake craniotomy; G, craniotomy under general anesthesia. Sampling times were as follows: T1, immediately after arterial line insertion; T2, immediately after head fixation in head frame; T3, $1 \mathrm{~h}$ after start of incision; T4, immediately after relief of head fixation. In $\mathrm{T} 1$ to $\mathrm{T} 4$, plasma levels of adrenaline were significantly higher in awake craniotomy than in craniotomy under general anesthesia $(\mathrm{P}<0.05)$.

\section{Results}

Plasma levels of adrenaline were significantly higher in awake craniotomy than in craniotomy under general anesthesia at T1-T4 according to the Wilcoxon rank-sum test $(\mathrm{p}<0.05)$ (Figure 1, Table 1). On the other hand, no difference in plasma levels of adrenaline was 
Page 3 of 5

seen between awake craniotomy and craniotomy under general anesthesia at T5 (Table 1).

Plasma levels of ACTH, cortisol, and noradrenaline were significantly higher for craniotomy under general anesthesia than for awake craniotomy at $\mathrm{T} 5$ using the Wilcoxon rank-sum test $(\mathrm{P}<0.05)$ (Figure 2, Table 1). Conversely, no significant differences in plasma levels of ACTH, cortisol, or noradrenaline were seen between awake craniotomy and craniotomy under general anesthesia at T1-T4 (Table 1). No significant difference in plasma levels of dopamine was seen between awake craniotomy and craniotomy under general anesthesia at any time points using the Wilcoxon rank-sum test (Table 1).

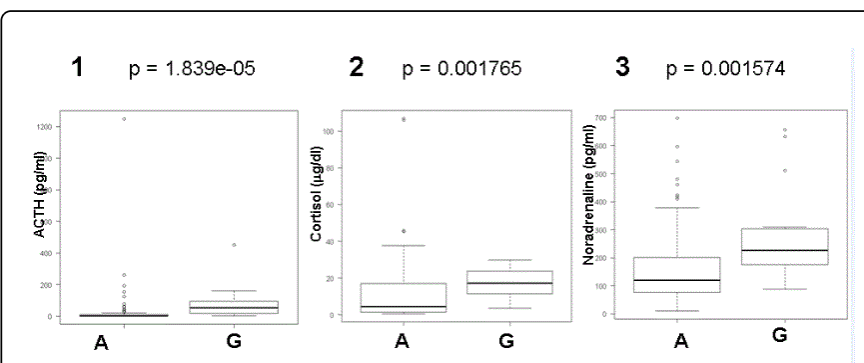

Figure 2: Comparison between plasma levels of ACTH, cortisol, and noradrenaline between awake craniotomy and craniotomy under general anesthesia Plasma levels of ACTH (panel 1), cortisol (panel 2), and noradrenaline (panel 3) in awake craniotomy and craniotomy under general anesthesia were compared using the Wilcoxon rank-sum test. A, awake craniotomy; G, craniotomy under general anesthesia. In T5 (immediately after arrival in the intensive care unit), plasma levels of $\mathrm{ACTH}$, cortisol, and noradrenaline were significantly higher in craniotomy under general anesthesia than in awake craniotomy $(\mathrm{P}<0.05)$.

\begin{tabular}{|l|l|l|}
\hline $\begin{array}{l}\text { Stress } \\
\text { hormones }\end{array}$ & Awake & General anesthesia \\
\hline Adrenaline 1\# & $170 \pm 353$ & $33 \pm 61$ \\
\hline Adrenaline 2\# & $822 \pm 416$ & $74 \pm 147$ \\
\hline Adrenaline 3\# & $194 \pm 124$ & $68 \pm 58$ \\
\hline Adrenaline 4 & $88 \pm 93$ & $51 \pm 38$ \\
\hline Adrenaline 5 & $82 \pm 97$ & $121 \pm 117$ \\
\hline ACTH1 & $21 \pm 25$ & $14 \pm 9$ \\
\hline ACTH2 & $18 \pm 40$ & $11 \pm 7$ \\
\hline ACTH3 & $36 \pm 73$ & $25 \pm 78$ \\
\hline ACTH4 & $24 \pm 123$ & $5 \pm 8$ \\
\hline ACTH5\# & $25 \pm 124$ & $84 \pm 113$ \\
\hline Cortisol1 & $11 \pm 8$ & $11 \pm 4$ \\
\hline Cortisol2 & $10 \pm 9$ & $9 \pm 3$ \\
\hline Cortisol3 & $16 \pm 43$ & $8 \pm 4$ \\
\hline Cortisol4 & $11 \pm 20$ & $4 \pm 4$ \\
\hline Cortisol5\# & $11 \pm 17$ & $18 \pm 9$ \\
\hline
\end{tabular}

This study found that plasma levels of adrenaline were significantly higher during awake craniotomy than during craniotomy under general anesthesia. The increased levels of adrenaline during awake craniotomy might agree with the increased heart rate and systolic arterial blood pressure seen during awake craniotomy [12]. On the other hand, plasma levels of ACTH, cortisol and noradrenaline were significantly higher with craniotomy under general anesthesia than with awake craniotomy on arrival at the intensive care unit after surgery. Tacconi et al. [13] reported a similar result with postoperative cortisol levels lower in a group with awake video-assisted thoracoscopic surgery compared with a group under general anesthesia.

Several studies have reported patient perceptions of stress during awake craniotomy. According to a questionnaire assessing aspects of patient perceptions of the procedure, most patients tolerate awake craniotomy well [14]. Eighty-seven percent of patients reported feeling at ease during awake craniotomy, indicating high levels of patient satisfaction [15]. Scores for postoperative pain and physical disorders were likewise significantly lower in awake craniotomy than in craniotomy under general anesthesia [16]. On the other hand, awake surgery has led to postoperative psychological sequelae resembling posttraumatic stress disorder in some cases [17]. Although awake craniotomy is useful to preserve neurological function after surgery compared to craniotomy under general anesthesia, the risk of stress during awake surgery should be considered, because adrenaline levels 
were significantly increased during awake surgery compared to craniotomy under general anesthesia. However, increased levels of postoperative stress hormones after craniotomy under general anesthesia might be related to the high scores for postoperative pain and physical disorders seen using this method.

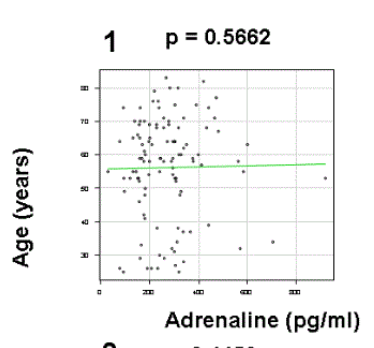

$3 \quad p=0.4459$

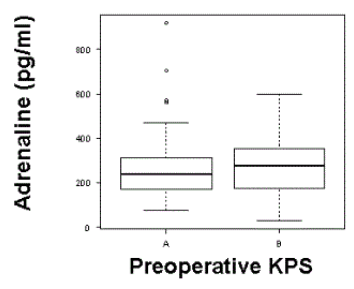

Figure 3: Correlations of mean adrenaline levels at 5 sample times with age, sex, 17 and Karnofsy Performance Scale before surgery, and neurological status after Surgery Correlations between mean plasma levels of adrenaline at 5 sample times and age, sex, Karnofsy Performance Scale (KPS) before surgery and neurological status after surgery were analyzed. No significant correlations were seen in all these studies. Panel 2: F, female; M, male. Panel 3: A, patients with KPS score $\geqq 80$ before surgery; B, patients with KPS score $<80$ before surgery. Panel 4: I, patients with improvement of neurological status after surgery; $\mathrm{D}$, patients with deterioration of neurological status after surgery; $S$, patients with same neurological status after surgery.

Why were adrenaline levels increased during awake craniotomy, while ACTH, cortisol and noradrenaline levels increased after craniotomy under general anesthesia? Several investigators have reported a dissociation between reactivity of the hypothalamuspituitary-adrenal axis and the sympathetic-adrenal-medullary system. In Alzheimer's disease and major depression, dissociation is seen between both systems $[18,19]$. Moreover, different responses to stress are exhibited by the hypothalamus-pituitary-adrenal axis and the sympathetic-adrenal-medullary system. The hypothalamus-pituitaryadrenal axis quickly habituates, while the sympathetic nervous system shows rather uniform activation patterns with repeated stress [20]. Since patients with brain tumors may well have experienced repeated episodes of stress, some degree of habituation might exist in the hypothalamus-pituitary-adrenal axis of patients undergoing awake craniotomy. On the other hand, stress levels of patients might be much higher just after craniotomy under general anesthesia than during awake craniotomy [6]. In addition, the differences in anesthetic drugs used between awake surgery and general anesthesia might also have contributed to the differences between systems. Further investigation is required to analyze discrepancies in the reactions of both systems.

As for biological markers of stress during awake craniotomy, Klimek et al. reported the inflammatory profiles of patients treated under awake craniotomy and craniotomy under general anesthesia to evaluate differences in surgical stress between groups, because surgical stress triggers inflammatory responses and releases mediators such as interleukins into human plasma [7]. They concluded that awake craniotomy does not cause any inflammatory response significantly different from that caused by craniotomy under general anesthesia. Moreover, they claimed that the visual analogue scale pain score was significantly lower in the awake group compared to the general anesthesia group at $12 \mathrm{~h}$ postoperatively. Similarly, Hol et al. (2009)[6] reported that awake craniotomy is likely to be physically and emotionally less stressful than general anesthesia after analyzing amino acid profiles to gain insights into physical and emotional stress.

Is the increase in adrenaline during awake craniotomy correlated to bad reactions of the brain or body induced by stress? Plasma levels of adrenaline did not correlate with neurological function, in terms of improvement, no change or deterioration after surgery in our study (Figure 3). This may be at least partly due to the blood-brain-barrier, which is impermeable to circulating adrenaline thanks to the protective influence of the locus ceruleus [21]. Moreover, vagal sensory fibers, directly activated by plasma adrenaline, represent the afferent limb of a negative feedback loop that adjusts the activity of the sympathoadrenal system [22]. Actually, in our study, increased plasma levels of adrenaline at T2 (fixation of head) had decreased rapidly by T3 ( $1 \mathrm{~h}$ after incision of the skin), a change that might be induced by negative feedback (Table 1).

In conclusion, plasma levels of adrenaline were significantly higher in awake craniotomy than in craniotomy under general anesthesia during surgery, while plasma levels of cortisol, ACTH and noradrenaline were significantly higher in craniotomy under general anesthesia than in awake craniotomy just after surgery.

\section{Acknowledgments}

This work was supported by the Japanese Foundation for Multidisciplinary Treatment of Cancer. We wish to thank Mr. Akira Saito for data analysis.

\section{References}

1. Pereira LC, Oliveira KM, L'Abbate GL, Sugai R, Ferreira JA, et al. (2009) Outcome of fully awake craniotomy for lesions near the eloquent cortex: analysis of a prospective surgical series of 79 supratentorial primary brain tumors with long follow-up. Acta Neurochir (Wien) 151: 1215-1230.

2. Sacko O, Lauwers-Cances V, BraUge D, Sesay M, Brenner A, et al. (2011) Awake craniotomy vs. surgery under general anesthesia for resection of supratentorial lesions. Neurosurgery 68:1192-1199.

3. Chacko AG, Thomas SG, Babu KS, Daniel RT, Chacko G, et al. (2013) Awake craniotomy and electrophysiological mapping for eloquent area tumours. Clin Neurol Neurosurg 115: 329-334.

4. Taylor MD, Bernstein M (1999) Awake craniotomy with brain mapping as the routine surgical approach to treating patients with supratentorial intraaxial tumors: a prospective trial of 200 cases. J Neurosurg 90: 35-41.

5. Peruzzi P, Bergese SD, Viloria A, Puente EG, Abdel-Rasoul M, et al. (2011) A retrospective cohort-matched comparison of conscious sedation versus general anesthesia for supratentorial glioma resection. Clinical article. J Neurosurg 114: 633-639.

6. Hol JW, Klimek M, van dre Heide-Mulder M, Stronks D, Vincent AJ, et al. (2009) Awake craniotomy induces fewer changes in the plasma amino acid profile than craniotomy under general anesthesia. J Neurosurg Anesthesiol 21: 98-107.

7. Klimek M, Hol JW, Wens S, Heijmans-Antonissen C, Niehof S, et al. (2009) Inflammatory profile of awake function-controlled craniotomy 
Citation: Shinoura N, Yamada R, Hatori K, Sato H, Kimura K (2014) Stress Hormone Levels in Awake Craniotomy and Craniotomy under General Anesthesia. J Neurol Neurophysiol 5: 256. doi:10.4172/2155-9562.1000256

Page 5 of 5

and craniotomy under general anesthesia. Mediators Inflamm 2009: 670480 .

8. Shinoura N, Yamada R, Kodama T, Suzuki Y, Takahashi M, et al. (2005) Preoperative fMRI, tractography and continuous task during awake surgery for maintenance of motor function following surgical resection of metastatic tumor spread to the primary motor area. Minim Invasive Neurosurg 48: 85-90.

9. Berger MS, Kincaid J, Ojemann GA, Lettich E (1989) Brain mapping techniques to maximize resection, safety, and seizure control in children with brain tumors. Neurosurgery 25: 786-792.

10. Dubois B, Slachevsky A, Litvan I, Pillon B (2000) The FAB: a Frontal Assessment Battery at bedside. Neurology 55: 1621-1626.

11. Kanda Y (2013) Investigation of the freely available easy-to-use software 'EZR' for medical statistics. Bone Marrow Transplant 48: 452-458.

12. Conte V, Guzzetti S, Porta A, Tobaldini E, Baratta P, et al. (2009) Spectral analysis of heart rate variability during asleep-awake craniotomy for tumor resection. J Neurosurg Anesthesiol 21: 242-247.

13. Tacconi F, Pompeo E, Sellitri F, Mineo TC (2010) Surgical stress hormones response is reduced after awake videothoracoscopy. Interact Cardiovasc Thorac Surg 10: 666-671.

14. Whittle IR, Midgley S, Georges H, Pringle AM, Taylor R (2005) Patient perceptions of "awake" brain tumour surgery. Acta Neurochir (Wien) 147: 275-277.

15. Wahab SS, Grundy PL, Weidmann C (2011) Patient experience and satisfaction with awake craniotomy for brain tumours. Br J Neurosurg 25: 606-613.
16. Wrede KH, Stieglitz LH, Fiferna A, Karst M, Gerganov VM, et al. (2011) Patient acceptance of awake craniotomy. Clin Neurol Neurosurg 113: $880-884$

17. Milian M, Luerding R, Ploppa A, Decker K, Psaras T, et al. (2013) "Imagine your neighbor mows the lawn": a pilot study of psychological sequelae due to awake craniotomy: clinical article. J Neurosurg 118: 1288-1295.

18. Pascualy M, Petrie EC, Brodkin K, Peskind ER, Wilkinson CW, et al. (2000) Hypothalamic pituitary adrenocortical and sympathetic nervous system responses to the cold pressor test in Alzheimer's disease. Biol Psychiatry 48: 247-254.

19. Ahrens T, Deuschle M, Krumm B, van der Pompe G, den Boer JA, et al. (2008) Pituitary-adrenal and sympathetic nervous system responses to stress in women remitted from recurrent major depression. Psychosom Med 70: 461-467.

20. Schommer NC, Hellhammer DH, Kirschbaum C (2003) Dissociation between reactivity of the hypothalamic-pituitary-adrenal axis and the sympathetic-adrenal-medullary system to repeated psychosocial stress. Psychosom Med 65: 450-460.

21. Harik S, McGunigal T Jr (1984) The protective influence of the locus ceruleus on the blood-brain barrier. Ann Neurol 15: 568-574.

22. Mravec B (2011) Role of catecholamine-induced activation of vagal afferent pathways in regulation of sympathoadrenal system activity: negative feedback loop of stress response. Endocvr Regul 45: 37-41. 